\title{
Interpretation of the Body Symbol of Northern Shaanxi Yangko*
}

\author{
Jiangcuo Zhaxi \\ School of Music and Performing Arts \\ Mianyang Teachers' College \\ Mianyang, China 621000
}

\begin{abstract}
The Northern Shaanxi Yangko culture originates from the northern Shaanxi region of China, it contains the national spirit and national emotions of Chinese nation. It is the most ethnically unique art form in northwest China. Northern Shaanxi Yangko is not only an art, but also a way to physically express the inner feelings of the dancer. As a body symbol, Northern Shaanxi Yangko not only expresses feelings through various body language, but also is a basic reflection of real life. The article interprets the body symbols of Northern Shaanxi Yangko, discusses the relationship between dance movements and dance language, as well as the synchronic and diachronic research directions and research methods of dance body symbols, and demonstrates the body language of the Northern Shaanxi Yangko in semiotics from dance movements and semantic changes; The topic of the study on the basis of theoretical research and empirical research to explore Northern Shaanxi Yangko, so it has important academic and practical significance.
\end{abstract}

Keywords-Northern Shaanxi Yangko; dance symbol; body language

\section{INTRODUCTION}

The Northern Shaanxi Yangko is a complex cultural system that covers many cultural issues and is inextricably linked with numerous traditional arts. It is the integration, intersection and formation of various types of arts, so it is an important part of Chinese culture and value system. It is the combination of culture and art folklore, it has a long history and with unique personality and fascinating charm. Northern Shaanxi Yangko accompanied the people of northern Shaanxi, it has different methods and cultural connotations. People here have no class, regardless of poverty or wealth, they all are the faithful inheritors and expressionists of Northern Shaanxi Yangko, and they all show the different inheritance and expression of Northern Shaanxi Yangko. This popular, national and regional dance style deeply rooted in folk, has become Northern Shaanxi Yangko and provides a fertile soil for the study of various art types.

Northern Shaanxi Yangko can be seen as a unique form of dance performance. It is a unified whole composed of body language. It is based on body language and expresses emotions and feelings through physical actions. The language of dance conveys the emotional changes of performers through each

*[Fund Project] This is the phased achievement of the 2017 Mianyang Social Science Union's Human Research Project "Culture Study of Body Dance and Dance Image ” (Project Number: MYSY2017QN01). movement and even subtle expression of the performer. Due to the influence of the creator's own emotions, regions, etc., the expression of content and emotion is also different: Some dances express inner feelings, some express interpretation of life, and others express group emotions, plus the differences in the thinking and personality of dancers, making a variety of dance forms, thus promoting the development of dance. For dance practitioners, dance is the most direct and convenient way to express daily feelings. It expresses emotions that are difficult to express in words through body language. As a typical representative of Chinese folk dance, Northern Shaanxi Yangko demonstrates its rich connotations through body language and promotes emotional communications. Therefore, the interpretation of the body symbol of Northern Shaanxi Yangko is indispensable in current academic world ${ }^{1}$. The article takes the body symbol of Northern Shaanxi Yangko as the research object, based on combing and summarizing historical documents and research results of previous generations, combining field investigations, it has conducted in-depth and multi-dimensional research on Northern Shaanxi Yangko, and in the meanwhile place the body language of Northern Shaanxi Yangko in the level of body language and linked with many parts of the body. It provides a reliable guarantee for the deep research of the Northern Shaanxi Yangko towards the background of a large culture and multidisciplinary three-dimensional crossover.

\section{THE TWO SIDES OF THE BODY SYMBOL OF NORTHERN SHAANXI YANGKO}

\section{A. Concept Definition}

From the origin, Northern Shaanxi Yangko is the integration of physical stretching exercises when people take rest after working. It is essentially an expression of the working mood of Northern Shaanxi people; through dance we can see the mood and image of northern Shaanxi people. The body symbol of Northern Shaanxi Yangko has its own dance language, which is the change of performer's body language. From a philosophical point of view, everything has its two sides, Northern Shaanxi Yangko is no exception.

Han Jing. Discussion on the role of body language in music major [J]. Music World. 2009.7 
On one hand, dance has certain advantages as a body symbol, through the dance language transmitted by the rhythm of the dancer's body, people's emotions can be expressed and exchanged. Whether it is the dancer's body movements or expressions such as eyes and face, it is a concrete expression of dance language. The dance language reflected by such movements and expressions is the most natural living language of people. Therefore, the dance symbol can also be the most authentic and accurate expression of the truest emotions of the northern Shaanxi people. Through the dance language of Northern Shaanxi Yangko, we can see the "first nature" of dance, which is the meaning of the original ecology. This also makes it easier for people to interpret the Northern Shaanxi Yangko in a more original ecological perspective. In different moods, people's dance language will express the corresponding emotions. For example, when the mood is sad, then the dance language is depressed, this can be manifested in the action of beating and it also can be expressed in the deep feelings of sadness and hidden bitterness. But whether it is action or facial expression, it is a series of dance language composed of a series of body symbols ${ }^{2}$. Another example is that many dance moves in Northern Shaanxi Yangko are direct stretching exercises after working. This can not only express emotions, but also can stretch muscles and stimulate the enthusiasm of labor. Therefore, when carrying out dance communication and education, if we could promote the Northern Shaanxi Yangko in the form of a body symbol, people will realize that the Northern Shaanxi Yangko is not only an expression of body language but also promotes physical health. So that they will make better understanding of the dance language and realize the importance of dance, this will make it easier to accept the Northern Song of Yangko, a body symbol that meets the aesthetic needs of people.

On the other hand, the dance of Northern Shaanxi Yangko as a body symbol also has certain drawbacks. The drawback lies in the contradiction between the dance movement language and human language itself. In dance, if we pay too much attention to the role of dance language in emotional communication, it will inevitably cause the deterioration of human language expression ability. Therefore, whether in the dance creation or interpretation, we must properly deal with the relationship between the two, the expression of the dance language must be accurate and appropriate, and advocate the use of language expression to carry out emotional exchanges in order to avoid the deterioration of people's language expression ability .

\section{B. Relationship Structure}

As a body symbol, Northern Shaanxi Yangko is composed of "dance movement" and "dance language". Northern Shaanxi Yangko expresses working people's mood and labor image through the content of labor dance. Therefore, Northern Shaanxi Yangko is also composed of its own dance movement and dance language.

Guo Lanlan. The Role of Body Language and Sentences in Music Education[J]. Journal of Beijing Normal University (Humanities and Social Sciences) .2001.5.
Dance movements often appear in the form of movement combination. This combination of movements can be divided into simple physical movements and emotional dance movements. The form of simple physical movements is relatively simple, it is basically a combination of people's familiar upper limb movements and simple spinning and leaping dance movements. This kind of dance has a simple connotation and rarely involves the expression of complex emotions; Emotional dance movements need to have more complex dance movements, but also it should combine with people's emotional factors, such as when expressing joyous dances, they should both have cheerful dance movements and happy facial expressions. They must express their labor actions through dance movements, but also reflects the positive attitude and emotions. The emotional dance movements will make Northern Shaanxi Yangko more infectious, and it could better reflect the dance movement is an important part of dance this human body symbol. ${ }^{3}$

Dance language is the sum of dance body language that expresses emotions. The composition of dance language is more complex, including dance movements, as well as the dance composition and the scenes of life it represents. Simple dance movements are unable to express the dance language, only the dance movements that incorporate emotional expression, spiritual transmission, and depiction of life scenes are the real dance language. Due to people's emotions, dance language has a strong appeal. Through the dance language, we can see the most primitive and accurate interpretation of the body symbol of Northern Shaanxi Yangko.

\section{THE SYNCHRONIC AND DIACHRONIC RESEARCH AND SIGNIFICANCE OF THE BODY SYMBOL OF NORTHERN SHAANXI YANGKO}

\section{A. Two Research Directions of Northern Shaanxi Yangko}

Northern Shaanxi Yangko is a kind of comprehensive art form. The current academic circles use synchronic and historic two directions to fully understand the dance body symbols.

The synchronic study of body symbols in Northern Shaanxi Yangko focused on the overall expression of dance. The Northern Shaanxi Yangko has been produced in people's social practice. Since its formation and development, it has undergone great changes and developments both in dance movement and dance language. As the sum of a series of actions expressed as body language mainly expresses inner feelings and reflects life through movements such as the eyes, crotch, knees and feet. At the same time, in the expression of body language, it is through dance performance, gestures, expressions, composition, and other expressions to form aesthetically pleasing dance movements, in order to create concrete images, and to express the inner feelings of dance images through dance movements and dance language.

The diachronic study of the body symbol of Northern Shaanxi Yangko focuses on the historical development

\footnotetext{
3 Zhao Fang. Talking about the Influence of Dance Learning on the All-round Development of Students' Quality [J]. Journal of Liaoning Normal College (Social Science Edition). 2002.4.
} 
situation from its birth to its development. As a body symbol, the Northern Shaanxi Yangko is used to express the labor scene at the beginning, use this carrier to demonstrate the inner joy. With the development of society, both the form and the content have undergone tremendous changes. The dance creators have added some typical facial expressions or movements in it, which on one hand has made the Northern Shaanxi Yangko livelier and more appealing, on the other hand it verified and interpreted the conclusion that Northern Shaanxi Yangko is a body symbol.

\section{B. Diversity in the Overall Study of Northern Shaanxi Yangko}

For the argumentation of the conclusion that "Northern Shaanxi Yangko is a body symbol", the academic community adopts a variety of research methods in both synchronic and diachronic directions. Among which the mainly and most important method is the historical literature research method and empirical inspection method.

On one hand, the study of dance in the academic world is based on examining a large amount of historical literature and through the research of labor patterns and labor achievements of each period to find arguments that can support the conclusion that Northern Shaanxi Yangko is a body symbol. Specific to Yangko this dance form, the origin of Yangko in China can be traced back to the Southern Song Dynasty. In the book "Xin Nian Za Yong Chao", Wu Xilin believed that "Yangko, the village fun of Southern Song Dynasty", from which we could know that there is a long history of Yangko in northern Shaanxi Province, at the same time, Yangko is produced in the land of labor, that is, the field. The relevant discussion can also be seen from Li Diaoyuan's "Yue Dong Bi Ji", he said "Farmers, in the late spring, dozens of women and children planted rice in the field, there is a drum on the side, when the drum was beaten, the farmers will make group labor, the drum sound will last the whole day, which we called it Yangko". " The birth of Yangko is closely related to the working people and is a product of working people during labor practices. "It was said that the Yangko originates from the Song of Rice Transplantation in Southern place, which belongs to the people sang for what they've done". 5 Through the literature, we can see that the Northern Shaanxi Yanhko was originated in Northern Shaanxi Province of China and it was created by the working people of northern Shaanxi region during their labor practices. It is one of the most ethnically distinctive dance forms in China, and it has a relatively high level of popularity and a good mass base in northern Shaanxi region.

On the other hand, for the study of Northern Shaanxi Yangko, researchers also need to perform a lot of dance observations in person, and even need to go deep into the front line of labor in northern Shaanxi region, and make filed research on the inheritance and development status of Northern Shaanxi Yangko.

\footnotetext{
Li Diaoyuan. Yue Dong Bi Ji [M]. Shanghai Huiwentang, 1915:320.
} Ding Yibo. Exploration of Yangko [J]. Xungen, 2001: (2): 76.
Both synchronic and diachronic researches require empirical data to verify that dance is a body symbol. Only by combining historical literature research and empirical research can we better interpret Northern Shaanxi Yangko and better promote its innovation and development. ${ }^{6}$

\section{THE BODY LANGUAGE OF NORTHERN SHAANXI YANGKO IN SEMIOTICS}

China is a unified multi-ethnic country, and because of its regional and national character, dance languages of various ethnic groups are different. As a unique dance form in northern Shaanxi, Northern Shaanxi Yangko has special dance body language. Its movements such as hands, feet, body and head are all different from other ethnic dances. To fully understand the dance language of Northern Shaanxi Yangko, we need to interpret it from all levels.

\section{A. The Body Movement Requirements of Northern Shaanxi Yangko}

1) Hand: Hand movement is one of the most basic dance movements. Different from the labor dance of Northeast Yangko and minority cultures, Northern Shaanxi Yangko focuses on to express the special emotions of the northern Shaanxi people through special hand movements and body language, thus reflecting dance information and ethnic culture.

Northern Shaanxi Yangko originated from the labor practices of the people in northern Shaanxi. It was the creation of working people during the long-term labor practices. As one of the important forms of "local specialties" traditional dance of the Han nationality, Northern Shaanxi Yangko requires the coordination of the upper limbs, feet and wrists, among which the basic hand position is very complicated. There are mainly three kinds of basic hand positions in Northern Shaanxi Yangko, the first kind of basic hand position is the akimbo position, this hand position requires the performer to hold the towels tightly. The second hand position is to place thumbs on both sides of the waist while holding the towels, the third position is when the musical melody begins to perform, one must buckle his entire elbow to his own body to show a happy and laboring atmosphere. These three hand positions perfectly present the cheerful labor scene of the people in northern Shaanxi and use flexible hands to demonstrate the passionate and labor-driven dance. This is also the essence of the Han labor dance.

Cultural development requires exchanges and learning. In the process of continuation and development, Northern Shaanxi Yangko learns and absorbs the essence of other minority dances and Western dances. Whether it is Han nationality dance, a minority dance, or Western dance, they all attach importance to the role of hand movements in dance performance. When performing the Northern Shaanxi Yangko, it is necessary to have agility in the hand movement, but also it need to pay attention to the coordination and communicability with the changing rhythm, and to show dance skills and labor behavior by changing the hand movement patterns. At the

Shi Jingxin. The narrative exploration of the body language of Chinese dance drama [J]. Journal of Beijing Dance Academy 2006(01) 
same time, dance performances in Northern Shaanxi Yangko also need to pay attention to balance and hand strength, such as the Panzi dance in Northern Shaanxi Yangko, which requires the performer to perform with knife prop. In this way, when performing Northern Shaanxi Yangko, it is necessary to not only maintain the balance of props, but also to pay attention to the coordination of the hand movements and the overall movement. This requires the superb balance and strong hand strength of the performers. ${ }^{7}$

2) Feet: Walking is the most basic movement of human beings and it is also one of the most basic movements of dance. Through the decomposition and interpretation of the feet movements in Northern Shaanxi Yangko, it could help us to have a more comprehensive understanding of Northern Shaanxi Yangko, and also we could better understand the dance language in it.

Like the hand position of hand movements, the feet movements of Northern Shaanxi Yangko also have basic feet positions. For example, when expressing cheerful labor emotions, the feet requirement of Northern Shaanxi Yangko is to press the heel in a positive position and perform regular rotation and dance under this position. Northern Shaanxi Yangko has excited melody and fast dance rhythm, so it has more downbeat, which requires performer to have a high degree of knee power. When performing the downbeat of Northern Shaanxi Yangko, the feet must drop at downbeat, and it is necessary to ensure that the feet do not stand upright too high, standing too high is not conducive to ensuring a stable center of gravity and physical balance. Dance rotation in the lower position can better control the balance of the body and the smoothness of the rotation, so as to better express the joy of the working people.

3) Body: As an art form of body symbol, the body's important position in Northern Shaanxi Yangko performance does not need to say more. Northern Shaanxi Yangko is derived from the labor practices of the people in northern Shaanxi. In labor, the use frequency of body is the highest. Northern Shaanxi Yangko is a manifestation of the emotional externalization of the working people in northern Shaanxi. To better perform the Northern Shaanxi Yangko, you need to combine upper body rhythm and lower body rhythm, so that you can express the original meaning and inner emotions of the Northern Shaanxi Yangko. When performing Northern Shaanxi Yangko, in order to make the body move up and down, attention must be focused on the waist. The waist is the body part that connects the upper body and the lower body and is the key point to drive the upper and lower body to dance. When performing Northern Shaanxi Yangko, it is necessary to use the waist as the force point, and use the power of the entire waist to drive the waist to perform infinite loop of arc drawing. The swing of the waist drives the regular rhythm of the upper body, with the aid of the waist strength, it is possible to perform elbow swinging and other dance movements at the same time, so that the body's upper body

Liu Jian. The Symbol of Silent World- Dance Body Language [J]. Journal of Beijing Dance Academy. 2000 (01). alternately rotates could express the warm, optimistic, and hard-working labor image of the working people in northern Shaanxi.

4) Head: Generally speaking, as the body symbol of Northern Shaanxi Yangko, it requires coordination from the head, shoulder, waist, arms and even eyes. Therefore, the head which is responsible for the transmission of eyes and neck movements also plays an extremely important role in the body language of Northern Shaanxi Yangko.

The keynote of Northern Shaanxi Yangko is generally nifty and lively, emphasis on the combination of rigidity and softness to express the joyful working mood of the laborers. There are many head movements in Northern Shaanxi Yangko. Take the common head shaking movement as an example, the general Northern Shaanxi Yangko will have head shaking movements, with head raise, head lift, head bow and high frequency head shaking movements to present the laborers sweat sweeping, weeding with lower head and other hard working movements. This dance movement, which originates from life and shows real life scenes, has a strong appeal and enables viewers to better appreciate the great image of the hard working people with enthusiasm.

Northern Shaanxi folk songs are an important embodiment of the broad and profound Chinese culture. In Northern Shaanxi Yangko incorporates the customs and inner feelings of the northern Shaanxi region. Through dance movements, not only can we demonstrate the labor scene, but we can also appreciate the national feelings. In Northern Shaanxi Yangko dance, the head movements can show the image of the working people and the labor scene with specific dance movements. They can also express the feelings of the workers through performer's facial expression changes. Take the representative head movement Shuanghutou in Northern Shaanxi Yangko as an example, when performing Shuanghutou, the dancers' arms must be raised on both sides of the body at the same time and the towels should be held overhead. When raising the towels, the head should swing from left to right, and when the towels are lifted to the top of the head, they should make towel flowers on both sides of the head. In particular, it is important to ensure high-frequency swing movements are performed when doing Baitou movements, so that the working people's high emotions are incorporated into them. When doing Shuanghutou, the face should always maintain a happy mood, eyes must open large and agile in order to make a pleasant smile. Raise the towels, make flower around the head, high-frequency body swing, the joyful and cheerful look made the dance performances of Northern Shaanxi Yangko more vivid. They can show the labor image and cheerful mood of laborers in different periods, and show unique personality and ethnic style of working people in the northern Shaanxi area with Yangko dance. ${ }^{8}$

In summary, as an artistic expression, Northern Shaanxi Yangko mainly expresses its subject and connotation through body language. Through the interpretation of the hand, feet, body, and head movements in Northern Shaanxi Yangko, it can better help us understand the body language of Northern $2011(10)]$
Wang Shaocan, Guo Chao. Body language in singing [J]. Yi Hai. 
Shaanxi Yangko. Through the dance language projected by the dance movements of various parts, we can fully understand Northern Shaanxi Yangko this national essence, understand the hard-working labor scene of the people in Northern Shaanxi, understand the national character and working mood of the working people, so that to help us to make better promotion of the all-round development of Northern Shaanxi Yangko.

\section{B. Body Language and Semantic Expression of Northern Shaanxi Yangko}

The semantic expression of dance needs to use the human body as a material carrier. Northern Shaanxi Yangko uses the body language contained in the dancer's body movements to express the semantic of the dance itself, and the change of dancer's posture, movement, number, and position all affect the dance's semantic changes.

1) Semantic changes caused by changes in body language and movement: During the performance of Northern Shaanxi Yangko, the change of speed, intensity, or radian of dance moves will change the original dance meaning. This change is precisely the charm of dance, not only it brings new artistic experience to the audience, but also brings more inspiration to dance creators, and is conducive to advancing the reform and innovation of dance.

From the body language of Northern Shaanxi Yangko, we can use the upper body, crotch, and legs to interpret the semantic changes caused by changes in speed, strength, or radian in body movements of Northern Shaanxi Yangko.

First, the semantic changes brought by changes in the upper body movements. During the performance of Northern Shaanxi Yangko, it is mainly use the axial rotation of the waists to drive arm swing and in conjunction with the footstep changes, it becomes a whole performance. In the slow-paced Northern Shaanxi Yangko, with the rhythmic slow pace of the footsteps, the dancer's body swings scope is small, and the arm swings scope is big, so the graceful posture of the dancer is demonstrated and presented to the audience a quiet and elegant work break scene. However, in a fast-paced performance, the dancers stepped at a fast pace, and the large, rapid swing of the waist speed up the movements of arms and upper body. Due to the faster body movements, the swing scope of the arm is small but the rhythm is brisk, so that to demonstrate a cheerful working mood with lively and jumping postures; second, the semantic changes brought by the changes in the crotch movements. The crotch movement in Northern Shaanxi Yangko is also a way for the dancer to convey emotions. The crotch movement in Northern Shaanxi Yangko is mainly based on one leg and twists or shakes the crotch with a small but light movement. The use of crotch movement can both vent the dancer's emotions and also enhance the infection of Northern Shaanxi Yangko, so it is easier to push the performance to a climax. However, the crotch movements with different pace have different semantic meanings, under soothing music the crotch movements are soft and soothing so that to present the body lines of the dancers and convey the unique beauty of body language. Under fast pace, the dancers' crotch movements have small radiant but faster, the upper body and arm also swing rapidly along with the fast-paced crotch movement, which give the audience a stronger visual impact and completely demonstrate the joyful labor scene of the people in Northern Shaanxi; Third, the semantic changes brought by changes in leg movements. The leg movements of Northern Shaanxi Yangko are distinctive. The most representative leg movement in Northern Shaanxi Yangko is the large-step movement which the lower leg was driven by upper leg. During the performance of Northern Shaanxi Yangko, the change of speed, strength, and radiant in the leg movement convey the different semantic information of dancer. The slow leg movements depict quiet work breaks time, while the fast paced big steps represents the free and joyful feeling of the people in northern Shaanxi. The northern Shaanxi people lived in the Loess Plateau with hard natural conditions for generations, the big step leg movement is more able to show their optimistic life status and the cheerful mood of celebrating the harvest.

2) Different semantic meanings conveyed by the change of the number of dancers in body language: Dance is an art form performed by dancers and dancer is the subject of dance performances. As a kind of collective dance, when the number of subjects in Northern Shaanxi Yangko changes, the semantic meaning of collective dance will change significantly.

During the Northern Shaanxi Yangko performance, different atmospheres were rendered due to the number of performers. In general, the dance atmosphere is in proportion to the number of performers. The more people there are, the more active the performance atmosphere will be, and the more infectious it will be. Under the influence of this powerful infectivity, more and people are attracted into the dance. Northern Shaanxi Yangko is a typical collective dance, especially when it takes a lot of people to participate in largescale performances. Therefore, we often have such experience when performing Yangko: a few or dozens of people dance at the same time would make the performing atmosphere gradually increased, and with the number of dancers changing from a few to several dozen, the atmosphere of the scene tends to culminate quickly. Especially in the large-scale Yangko performances, the open terrain often attract hundreds of people participating in the performance, every audience on the scene are infected with this cheerful atmosphere. It is for this reason that the large number of participants has become a major feature of Northern Shaanxi Yangko performance. In largescale festivals, the director will also make full use of a large number of actors to create this atmosphere, such as the waist drum performances during the military parade of the 60th anniversary of the founding of the PRC. The large number of people can not only enrich the form of Northern Shaanxi Yangko, but also receive better visual effects due to changes in the color of the props and costumes.

In addition, in Northern Shaanxi Yangko dance, there is a dance form called "Duiwu" which is a collective participation dance form. The aforementioned waist drum is also a typical dance that expresses love between men and women. There are a large number of participants in Duiwu, and these participants are divided into relatives of men and women. There is a special connotation in Duiwu, during the performance the combination of men and women could better show love and life interest, if 
the sex ratio of male and female gender is imbalanced, the side with more people tends to show themselves more in order to attract attention. At this time, the body language of dance will be more unrestrained and exaggerated, with more expressiveness and tension, and to a certain extent, it also reflects the human nature of primitive sex chase. When the number of participants is small, since the number of dances cannot fully mobilize the atmosphere of the scene, the semantics will be relatively vague at that time. When the number of dance pairs increases, the performance atmosphere will rise, and the dancers' emotions will be fully mobilized. The speed, strength and radiant of the movement will increase accordingly, so the dancers' body language will have a clearer direction.

3) Body hint of body language during dancer's body position change: Dance performances are often performed on the stage, and the audience can only appreciate it from far away, which opens up the distance between the audience and the dancers. Due to distance restrictions, dancers often only have limited interaction with the dancers through applause, and the contents reflected by the dancer's body language could only be interpreted passively. In Northern Shaanxi Yangko performance, different postures of dancers convey different semantic meanings. Different from the dance that performed on stage, the folk Northern Shaanxi Yangko performances are often presented in circle dance style. During the circle dance performance, dancers are often surrounded by the audience. Due to the need to focus on the dance content, the dancers often stand opposite to each other hand in hand and the dancers are very close to the audience. The Northern Shaanxi Yangko which is very close to people's lives, the performer may be familiar to the audience. It is precisely because of this acquaintance relationship so that when the dancers perform Yangko dances such as love themes, they will care more about the views of the surrounding audiences, especially audiences that know them. Under this psychological effect, dancers often make deformation movements due to psychological changes, such as physical tension, rigid movements, etc. At this time, body language deviates from the original meaning of dance. Because of the close distance, audience can have direct communication with dance performers, and such communication often have the most direct influence on dancers. For example, during the performance of "Waist drum", when entering the Duiwu dance, sometimes when one party wants to be opposed to the other and the other avoid with dance, the crowd often burst into friendly laughter or shouting. Such friendly acts could bring pressure to the dancers and cause deformation movements, or they may also stimulate the dancers to respond with more exaggerated and more intense dance movements.

In addition, factors that caused the semantic change of Northern Shaanxi Yangko also include cultural and environmental factor which brought by regional characteristics and science and technology information. On one hand, Northern Shaanxi Yangko must maintain its own national characteristics, on the other hand, it must not be self-contained, it should actively use contemporary technology to communicate and spread. Although modern technology will to some extent eliminate the national characteristics of Northern Shaanxi Yangko, art forms that are out of touch with the times and do not have characteristics of the times are difficult to survive. Under the background of the times, Northern Shaanxi Yangko will only gradually innovate after being baptized with modern science and technology and actively adapt to social development, only in this way can it achieve its own better inheritance and development. In spite of this change, the semantics of body language in Northern Shaanxi Yangko will also change accordingly, however, this change is neither backward nor perishable, and it is the newborn of Northern Shaanxi Yangko that is both ethnic and time-honored.

\section{CONCLUSION}

In the current world of globalization, modernization, and economic integration, the deepening of the humanities and arts, and further strengthening the exploration of Northern Shaanxi Yangko's inheritance and development, its significance not only for China but also for the world. It not only fully affirms the historical value and contemporary significance of traditional culture, but also realizes creative transformation and innovative development of traditional culture, giving it new generational connotation and modern expression form, enabling it to gain new vitality and making excellent traditional culture become the source to conserve a new generation's value, it is also the mission of every humanist. Dance as an art form comes from people's long-term social practice. Northern Shaanxi Yangko is the most representative art form in northern Shaanxi region. It was born from the great practice of the northern Shaanxi people, and reflects the inner feelings of the northern Shaanxi people and their longing for labor and life. It is an important representative of traditional Chinese culture.

We should clearly see that the study of body symbols of Northern Shaanxi Yangko is precisely one of the efforts to build along the research. Only let more people understand and pay attention to the study of Northern Shaanxi Yangko, it could make better development. We can further and systematically deepen our understanding of Northern Shaanxi Yangko and make a strong contribution to the further deepening and improvement of Northern Shaanxi Yangko. Any person who contributes to the culture and art of Northern Shaanxi Yangko will have a happy life.

\section{REFERENCES}

[1] Zhang Suqin. Study on the nature of dance body language [J]. Journal of Beijing Dance Academy. 2011(01). 张素琴.舞蹈身体语言的本质研究 [J]. 北京舞蹈学院学报. 2011(01)

[2] Liu Jian, Pan Duoling. The body language of dance - stage scheduling [J]. Journal of Jilin College of Arts. 2009 (01). 刘建,潘多玲.舞蹈身体 的群言—舞台调度[J]. 吉林艺术学院学报. 2009(01)

[3] Peng Jixiang, Lv Yisheng, Ping Xin. Talk about dance aesthetics [J]. Journal of Beijing Dance Academy. 2009 (01). 彭吉象, 吕艺生, 平心.舞 蹈美学漫谈[J]. 北京舞蹈学院学报. 2009(01)

[4] Wang Bin. International dance academic trends [J]. Journal of Beijing Dance Academy. 2009 (02). 王涁.国际舞蹈学术动态[J]. 北京舞蹈学 院学报. 2009(02) 
[5] Zhaxi Jiangcuo. Discussion on the Inheritance and Development of Northern Shaanxi Yangko[J]. Journal of Yan'an University (Social Science Edition). 2006(01). 扎西江措. 论陕北秧歌的传承发展 $[\mathrm{J}]$. 延安 大学学报(社会科学版). 2006(01)

[6] Peng Jixiang, Ping Xin, Tang Xumei, Han Qiaobing, Art and Dance Development: The Art Psychology and Dance Psychology [J]. Journal of Beijing Dance Academy. 2008 (01). 彭吉象, 平心, 汤旭梅, 韩养冰 艺 术学与舞蹈学发凡——兼谈艺术心理学和舞蹈心理学 [J]. 北京舞蹈 学院学报. 2008(01)

[7] Li Jieming. The Emotion of Dance [J]. Journal of Beijing Dance Academy. 2008 (02). 李杰明.舞蹈的情感 [J]. 北京舞蹈学院学报. 2008(02)

[8] Liu Jian. When Dance is Culture and Art [J]. Journal of Beijing Dance Academy. 2007 (01). 刘建. 当舞蹈作为文化和艺术时 [J]. 北京舞蹈学 院学报. 2007(01)

[9] Yang Lan. The development of physical expression and the opportunity for the rise of dance aesthetics [J]. Journal of Beijing Dance Academy. 2007 (02). 杨岗.身体表现力的发展与舞蹈美学兴起的契机[J]. 北京 舞蹈学院学报. 2007(02) 